Musées, Patrimoine et Culture scientifiques et techniques

177 | 2018

mai-juin 2018

\title{
Responsable de Fab Lab : un facilitateur numérique
}

\section{Guillaume Carpentier et Serge Lochot}

\section{OpenEdition \\ Journals}

Édition électronique

URL : http://journals.openedition.org/ocim/2583

DOI : $10.4000 /$ ocim. 2583

ISSN : 2108-646X

Éditeur

OCIM

Édition imprimée

Date de publication : 1 mai 2018

Pagination : $42-43$

ISSN : 0994-1908

Référence électronique

Guillaume Carpentier et Serge Lochot, «Responsable de Fab Lab : un facilitateur numérique », La Lettre de l'OCIM [En ligne], 177 | 2018, mis en ligne le 01 mai 2019, consulté le 05 juin 2019. URL: http://journals.openedition.org/ocim/2583 ; DOI : 10.4000/ocim.2583

Ce document a été généré automatiquement le 5 juin 2019.

Tous droits réservés 


\section{Responsable de Fab Lab : un facilitateur numérique}

\section{Guillaume Carpentier et Serge Lochot}

\section{Un atelier au Fab Lab Kaléidoscope}

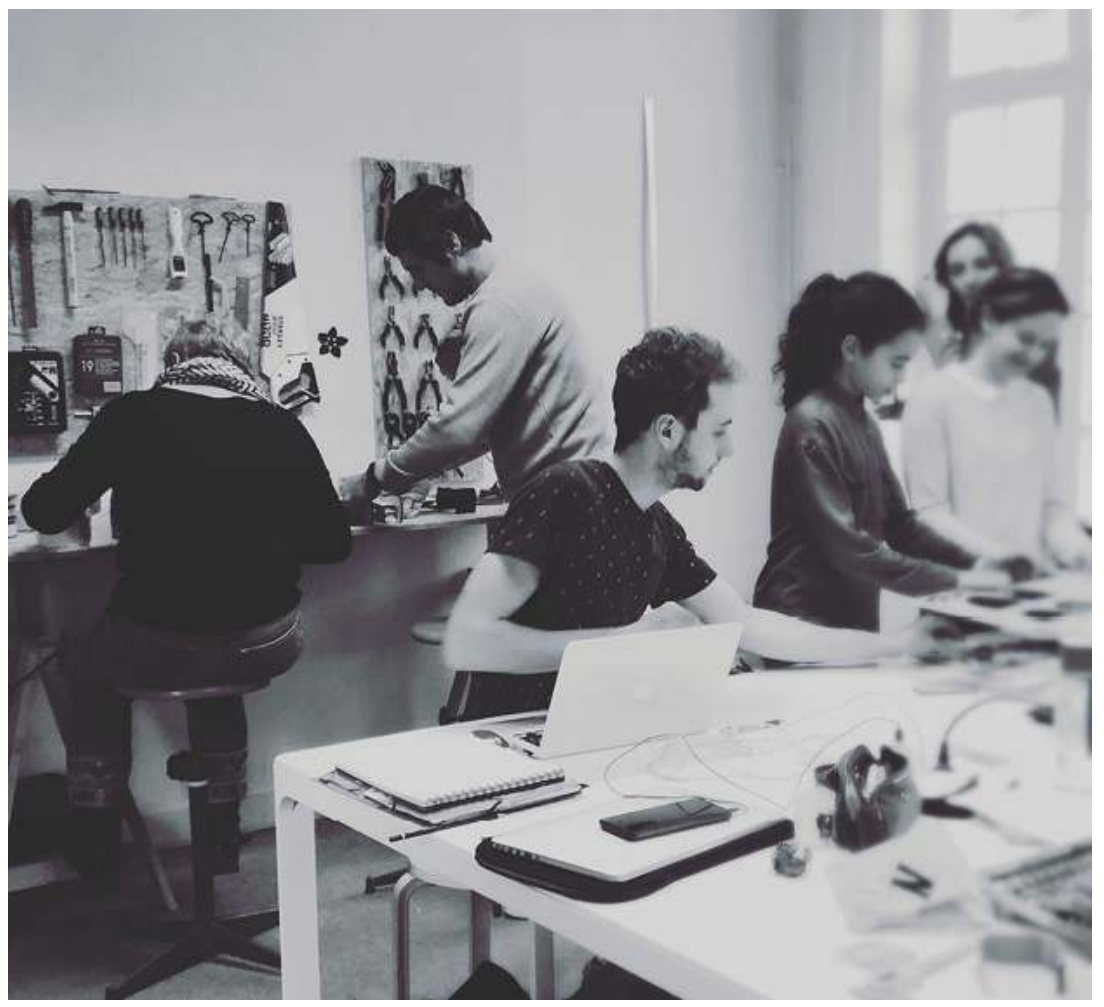

(c) Les Copeaux Numériques

Tout d'abord présentez-nous la structure dans laquelle vous travaillez.

Le Kaléidoscope est un lieu organisé autour du travail du bois et de la fabrication numérique situé à Petit-Quevilly (dans le périmètre de la Métropole Rouen Normandie). 
Géré par la coopérative (Scop) "Les Copeaux Numériques", il se déploie sur près de 1 $000 \mathrm{~m} 2$ autour d'espaces collaboratifs dédiés à l'artisanat, la création, la culture, les loisirs et l'entrepreneuriat social et solidaire où sont attachés à la structure un atelier de menuiserie traditionnel, un Fab Lab, des espaces de coworking, un café culturel.

Quel type de public fréquente le Fab Lab ?

Depuis son ouverture, en septembre 2017, la fréquentation est encourageante. Ce que nous observons pour l'instant, c'est qu'il n'y a pas a priori de public cible tant d'un point vue générationnel que social. Toutefois il faut nuancer ce constat car nous touchons quand même des bricoleurs, artistes, technophiles de tous profils confondus : homme/ femme, jeunes/retraités qui partagent une certaine ouverture et un esprit créatif... Il n'y a pas d'usager type, hormis une curiosité pour tout ce qui touche à la technologie numérique et à la création.

Y-a-t-il des activités organisées en direction des groupes scolaires?

Pas spécifiquement. Il existe simplement des ateliers réservés aux enfants entre 7 et 15 ans, mais qui ne sont pas organisés dans le cadre scolaire; c'est au bon vouloir de chacun et l'inscription est libre. Il s'agit d'ateliers thématiques au cours desquels les enfants découvrent les machines, les technologies et un peu de sciences. Nous avons par exemple organisé un atelier sur une voiture à air comprimé, en expliquant comment tout cela fonctionne. Nous avons également mis en place un atelier planeur pour comprendre pourquoi ces engins volent plus ou moins bien. Il y a là évidemment une dimension pédagogique de l'activité.

Comment vous définiriez-vous : facilitateur numérique, Fab Lab manager ou maker?

Pour moi le maker est une personne que l'on reçoit : le maker serait le nouveau bricoleur numérique. Je préfère le terme de facilitateur numérique, il correspond plus à la réalité de ma fonction qui consiste à mettre les visiteurs en relation avec le parc de machines, à les conseiller dans leur création. Je ne suis pas là pour faire à la place des autres mais plutôt pour montrer, aider, faciliter. Ensuite, chaque utilisateur réalise son projet comme il l'entend. Je ne suis pas là en prestataire.

Quel est votre parcours professionnel?

J'ai une formation de designer industriel, un cursus en quatre ans après le Bac qui mêle l'artistique et le technique notamment dans le domaine du design objet. J'ai ensuite eu l'opportunité de rencontrer les responsables de la scop "Les Copeaux numériques" alors qu'ils étaient au démarrage du projet. Ils cherchaient alors à créer à côté de l'espace dédié à la menuiserie un atelier numérique, un Fab Lab.

Quelle était votre motivation pour adhérer à ce projet de Fab Lab ?

Actuellement, j'exerce un métier de designer car je suis là pour mettre en forme les idées, tenter de résoudre les problèmes techniques, apporter les solutions. Ce qui est motivant et valorisant est lié au fait que je travaille non pour une entreprise mais pour une personne qui fabrique son propre objet. Par ailleurs, j'ai toujours eu une curiosité et un intérêt pour les imprimantes 3D, les logiciels de dessin 2D ou 3D et les multiples possibilités que ces outils offrent en matière de création.

Concrètement, en quoi consiste votre travail au Fab Lab ?

Il y a deux aspects. Le premier volet est la "formation" à l'utilisation et la pratique des logiciels et des machines, afin que chaque utilisateur puisse appréhender les possibilités technologiques des machines à commandes numériques mais également leurs limites. 
Et ainsi pouvoir les manipuler à bon escient. Le second volet est la partie "accompagnement" d'un point de vue individuel ou dans le cadre d'animation de groupe afin que chacun puisse travailler son projet individuellement ou collectivement. Car l'entraide entre les usagers est également importante. Les projets ainsi réalisés sont des plus divers : maquettes architecturales, sculptures, objets de décoration, bijoux ou encore éléments céramiques...

Le Fab Lab serait donc un tiers-lieu qui transforme en profondeur les manières d'apprendre et de transmettre les connaissances techniques?

Oui dans la mesure où dans les faits, nous abordons des projets de manière assez peu théorique et très vite pratique. Par exemple, pour un atelier sur la découpe laser, la partie théorique est certes nécessaire mais l'exercice pratique intervient rapidement dans le déroulement de la séance. Nous sommes davantage dans une démarche de coconstruction du savoir. Le modèle n'est pas celui, descendant, d'un expert qui délivre des connaissances. Il s'agit d'une vraie collaboration, d'un échange et d'un partage des logiciels et des savoirs de chacun, usager comme encadrant. Au fur et à mesure de la séance, les participants se conseillent mutuellement, font en sorte que personne ne prenne du retard. J'apprends également beaucoup des participants. L'échange est bénéfique dans les deux sens.

\section{L'aspect expérimental est également important?}

Les usagers ne viennent pas seulement pour utiliser les machines et appréhender des manières de faire codifiées, ils viennent principalement pour réaliser un objet et vivre une expérience singulière. Chaque projet est unique. Nous repartons de zéro à chaque fois avec cependant une base de connaissances réutilisables mais très souvent nous expérimentons, testons, recommençons, validons...

Lieu d'apprentissage collectif, le Fab Lab se défınit aussi par une dimension communautaire qui favorise le lien social?

Il s'agit là d'un aspect qui plaît particulièrement aux usagers : le fait de ne pas avoir en face de soi un professeur ou un référent. Ils apprécient beaucoup de montrer ce qu'ils sont en train de faire, de recueillir les avis et conseils de chacun... bref, de travailler et créer dans un univers bienveillant. Le côté coopératif du lieu est essentiel. Des projets en commun, non prévus au départ, émergent fréquemment de ces rencontres conviviales et ces échanges.

La revendication politique et militante n'est pas absente de l'univers des Fab Labs : il s'agirait d'aider la population à s'approprier une technologie et mettre à la disposition des citoyens les outils dont ils ont été dépossédés. Qu'en pensez-vous?

Effectivement les enjeux présents au départ du projet du Kaléidoscope étaient de redonner aux gens la possibilité de faire par eux-mêmes. Il s'agissait de leur offrir l'opportunité d'avoir accès à des machines numériques très performantes, polyvalentes - permettant d'obtenir des résultats de qualité professionnelle -, mais difficilement accessibles pour des raisons de coût. L'aspect mutualisation des savoirs et des machines est également déterminant dans la démarche et dans la construction de l'outil et son fonctionnement au quotidien. Ces enjeux restent plus que jamais fondamentaux. 


\section{RÉSUMÉS}

Interrogé sur ses pratiques et la manière de concevoir son métier, Guillaume Carpentier évoque la dimension collaborative des Fab Labs, le partage des savoirs qui s'y pratique à travers la mise en œuvre de dispositifs pédagogiques et sociaux inédits.

INDEX

Mots-clés : Fab Lab, réseau, CSTI

\section{AUTEURS}

\section{GUILLAUME CARPENTIER}

Responsable du Fab Lab Le Kaléidoscope au Petit-Quevilly

SERGE LOCHOT

Rédacteur en chef de La Lettre de l'Ocim 